\title{
Approximation properties of the modified Lupas-Kantorovich type operators
}

\author{
Bo-yong Lian * \\ ${ }^{1}$ Department of Mathematics, Yang-En University, Quanzhou 362014, P.R.China
}

\begin{abstract}
In this paper, the author introduce a class of modified Lupas-Kantorovich type operators which preserve constant and linear functions. By using modulus of continuity, modulus of smooth, K-functional and lipschitz class, the rate of convergence of these operators are derived. Finally, the author present a voronovskaya-type asymptotic formula.
\end{abstract}

\section{Introduction}

In the year 1987, Lupas [1] introduced PolyaBernstein operators

$$
P_{n}^{(1 / n)}(f, x)=\sum_{k=0}^{n} f\left(\frac{k}{n}\right) p_{n, k}^{(1 / n)}(x),
$$

where $f \in C[0,1], p_{n, k}^{(1 / n)}(x)=\frac{2(n !)}{(2 n) !}\left(\begin{array}{l}n \\ k\end{array}\right)(n x)_{k}(n-$ $n x)_{n-k},(n)_{k}=n(n+1) \cdots(n+k-1)$.

In [2], Miclaus studied some approximation properties of Bernstein-Stancu type operators based on Polya distribution. Recently, Gupta and Rassias [3] introduced the Durrmeyer variant of the operators (1) as follows:

$$
D_{n}^{(1 / n)}(f, x)=(n+1) \sum_{k=0}^{n} p_{n, k}^{(1 / n)}(x) \int_{0}^{1} p_{n, k}(t) f(t) d t,
$$

where $p_{n, k}(t)=\left(\begin{array}{c}n \\ k\end{array}\right) t^{k}(1-t)^{n-k}$. In[4], to approximate Lebesgue integrable functions, Agrawal introduced the following integral modification of the operators (1):

$$
D_{n}^{*(1 / n)}(f, x)=(n+1) \sum_{k=0}^{n} p_{n, k}^{(1 / n)}(x) \int_{\frac{k}{n+1}}^{\frac{k+1}{n+1}} f(t) d t .
$$

In 2003, King [5] introduced the modified Bernstein operators. Later, this idea was applied to some other well-known approximating operators,such as, the Szasz-Mirakjan operators [6], the Baskakov operators [7], the q-operators [8]. Obviously, these operators have a better rate of convergence than the classical operators.

Inspired by the idea of King, we propose the modified variant of the operators (3) in the following way:

$\widehat{D}_{n}^{(1 / n)}(f, x)=(n+1) \sum_{k=0}^{n} p_{n, k}^{(1 / n)}(r(x)) \int_{\frac{k}{n+1}}^{\frac{k+1}{n+1}} f(t) d t$,

where $r(x)=x+\frac{2 x-1}{2 n}, x \in\left[\frac{1}{4}, \frac{3}{4}\right]$.

The purpose of this paper is to study local approximation, pointwise estimates and global approximation results. Finally, we present a voronovskaya-type asymptotic formula.

We get some definition as follows:

Let $f(x) \in C[0,1], \delta>0$,

\section{Definition 1 (modulus of continuity)}

$$
\omega(f, \delta)=\sup _{0<h \leq \delta} \sup _{x, x+h \in[0,1]}|f(x+h)-f(x)|,
$$

^e-mail: lianboyong@163.com 
Definition 2 (the second order modulus of smoothness)

$$
\begin{gathered}
\omega_{2}(f, \sqrt{\delta})=\sup _{0<h \leq \sqrt{\delta}} \sup _{x, x+h, x+2 h \in[0,1]} \\
|f(x+2 h)-2 f(x+h)+f(x)|,
\end{gathered}
$$

\section{Definition 3 (K-functional)}

$$
K_{2}(f, \delta)=\inf _{g \in W^{2}}\left\{\|f-g\|+\delta\left\|g^{\prime \prime}\right\|\right\}
$$

where $W^{2}=\left\{g \in C[0,1]: g^{\prime}, g^{\prime \prime} \in C[0,1]\right\}$.

Definition 4 ( Lipschitz class)

$\operatorname{Lip}_{M}(\beta)=\left\{f|f \in C[0,1]| f,(t)-f(x)|\leq M| t-\left.x\right|^{\beta}\right\}$,

where $0<\beta \leq 1, M>0$.

Let $\phi(x)=\sqrt{x(1-x)}$ and $f \in C[0,1]$, the first order modulus of smoothness is given by [9]

$$
\omega_{\phi}(f, t)=\sup _{0<h \leq t}\left|f\left(x+\frac{h \phi(x)}{2}\right)-f\left(x-\frac{h \phi(x)}{2}\right)\right|,
$$

where $x \pm \frac{h \phi(x)}{2} \in[0,1]$.

Further, the corresponding K-functional to $\omega_{\phi}(f, t)$ is defined by

$$
K_{\phi}(f, t)=\inf _{g \in W_{\phi}[0,1]}\left\{\|f-g\|+t\left\|\phi g^{\prime}\right\|\right\}(t>0),
$$

where $W_{\phi}[0,1]=\left\{g: g \in A C[0,1],\left\|\phi g^{\prime}\right\|<\infty\right\}$ and $A C[0,1]$ is the class of all absolutely continuous functions on $[0,1]$.

\section{Some Lemmas}

We start this section with the following uesful lemmas, which will be used in the sequel.

Lemma 1(see [2]) For $e_{i}=t^{i}, i=0,1,2$, we have

$$
\begin{gathered}
P_{n}^{(1 / n)}\left(e_{0}, x\right)=1, P_{n}^{(1 / n)}\left(e_{1}, x\right)=x, \\
P_{n}^{(1 / n)}\left(e_{2}, x\right)=x^{2}+\frac{2 x(1-x)}{n+1} .
\end{gathered}
$$

Lemma 2(see [3]) For $e_{i}=t^{i}, i=0,1,2$, we have

$$
\begin{gathered}
D_{n}^{*(1 / n)}\left(e_{0}, x\right)=1, D_{n}^{*(1 / n)}\left(e_{1}, x\right)=\frac{2 n x+1}{2 n+2}, \\
D_{n}^{*(1 / n)}\left(e_{2}, x\right)=\frac{3 n^{3} x^{2}+9 n^{2} x-3 n^{2} x^{2}+3 n x+n+1}{3(n+1)^{3}} .
\end{gathered}
$$

Using the same method to lemma 2, we obtain

Lemma 3 For $e_{i}=t^{i}, i=0,1,2, x \in$ $\left[\frac{1}{4}, \frac{3}{4}\right]$, we have

$$
\begin{gathered}
\widehat{D}_{n}^{(1 / n)}\left(e_{0}, x\right)=1, \widehat{D}_{n}^{(1 / n)}\left(e_{1}, x\right)=x, \\
\widehat{D}_{n}^{(1 / n)}\left(e_{2}, x\right)=\frac{3 n^{3} r^{2}(x)+3 n^{2} r(x)(3-r(x))+3 n r(x)}{3(n+1)^{3}} \\
+\frac{1}{3(n+1)^{2}} .
\end{gathered}
$$

Remark 1 By simple applications of Lemma 3, we get

$$
\begin{gathered}
\widehat{D}_{n}^{(1 / n)}(t-x, x)=0, \\
\widehat{D}_{n}^{(1 / n)}\left((t-x)^{2}, x\right)=\frac{2 x(1-x)}{n}+O\left(n^{-2}\right)(n \rightarrow \infty) .
\end{gathered}
$$

$$
\widehat{D}_{n}^{(1 / n)}\left((t-x)^{4}, x\right)=O\left(n^{-2}\right)(n \rightarrow \infty) .
$$

Remark 2 When $n$ sufficient large, we have

$$
\widehat{D}_{n}^{(1 / n)}\left((t-x)^{2}, x\right) \leq \frac{3 x(1-x)}{n} .
$$

Lemma 4 For $f \in C[0,1], x \in\left[\frac{1}{4}, \frac{3}{4}\right]$, we have

$$
\left\|\widehat{D}_{n}^{(1 / n)}(f, x)\right\| \leq\|f\| .
$$

Proof From the definiton (4) and Lemma 3, we have

$$
\begin{aligned}
\left|\widehat{D}_{n}^{(1 / n)}(f, x)\right| & \leq(n+1) \sum_{k=0}^{n} p_{n, k}^{(1 / n)}(r(x)) \int_{\frac{k}{n+1}}^{\frac{k+1}{n+1}}|f(t)| d t \\
& \leq\|f\| \widehat{D}_{n}^{(1 / n)}(1, x)=\|f\| .
\end{aligned}
$$

Lemma 5(see[10]) For $f(x) \in C[0,1]$, then exists a constant $C>0$ such that

$$
K_{2}(f, \delta) \leq C \omega_{2}(f, \sqrt{\delta}) .
$$

Lemma 6(see[9]) For $f(x) \in C[0,1]$, then exists a constant $C>0$ such that

$$
K_{\phi}(f, t) \leq C \omega_{\phi}(f, t) .
$$




\section{Conclusion}

Theorem 1 For $f \in C[0,1], x \in\left[\frac{1}{4}, \frac{3}{4}\right]$, we have

$$
\lim _{n \rightarrow \infty} \widehat{D}_{n}^{(1 / n)}(f, x)=f(x) .
$$

Proof From the Lemma 3, we get

$$
\lim _{n \rightarrow \infty} \widehat{D}_{n}^{(1 / n)}\left(e_{i}, x\right)=x^{i}, i=0,1,2 .
$$

By Korovkin theorem, it follows that $\widehat{D}_{n}^{(1 / n)}(f, x) \rightarrow f(x)$ as $n \rightarrow \infty$.

Theorem 2 For $f, f^{\prime \prime} \in C[0,1], x \in\left[\frac{1}{4}, \frac{3}{4}\right]$, then

$$
\lim _{n \rightarrow \infty} n\left[\widehat{D}_{n}^{(1 / n)}(f, x)-f(x)\right]=x(1-x) f^{\prime \prime}(x) .
$$

Proof By Taylor's expansion, we may write

$$
\begin{aligned}
f(t)=f(x)+ & f^{\prime}(x)(t-x)+\frac{1}{2} f^{\prime \prime}(x)(t-x)^{2} \\
& +\phi(t ; x)(t-x)^{2},
\end{aligned}
$$

where $\phi(t ; x)$ is the Peano form of the remainder, $\phi(t ; x) \in C[0,1]$ and $\lim _{t \rightarrow x} \phi(t ; x)=0$.

By applying the operators $\widehat{D}_{n}^{(1 / n)}(f, x)$ to the above relation, we obtain

$$
\begin{gathered}
n\left[\widehat{D}_{n}^{(1 / n)}(f, x)-f(x)\right]=n f^{\prime}(x) \widehat{D}_{n}^{(1 / n)}(t-x, x)+ \\
\frac{n}{2} f^{\prime \prime}(x) \widehat{D}_{n}^{(1 / n)}\left((t-x)^{2}, x\right)+n \widehat{D}_{n}^{(1 / n)}\left(\phi(t ; x)(t-x)^{2}, x\right) .
\end{gathered}
$$

By the Cauchy-Schwartz inequality, we have

$$
\begin{gathered}
\widehat{D}_{n}^{(1 / n)}\left(\phi(t ; x)(t-x)^{2}, x\right) \\
\leq \sqrt{\widehat{D}_{n}^{(1 / n)}\left(\phi^{2}(t ; x), x\right)} \cdot \sqrt{\widehat{D}_{n}^{(1 / n)}\left((t-x)^{4}, x\right)} .
\end{gathered}
$$

Observe that $\phi^{2}(x ; x)=0, \phi^{2}(t ; x) \in C[0,1]$, then it follows from Theorem 1 that

$$
\lim _{n \rightarrow \infty} \widehat{D}_{n}^{(1 / n)}\left(\phi^{2}(t ; x), x\right)=\phi^{2}(x ; x)=0 .
$$

Now from (5), (6), (7), (10) and (11), we get immediately

$$
\lim _{n \rightarrow \infty} n f^{\prime}(x) \widehat{D}_{n}^{(1 / n)}(t-x, x)=0 .
$$

$$
\lim _{n \rightarrow \infty} n \widehat{D}_{n}^{(1 / n)}\left(\phi(t ; x)(t-x)^{2}, x\right)=0 .
$$

$$
\lim _{n \rightarrow \infty} \frac{n}{2} f^{\prime \prime}(x) \widehat{D}_{n}^{(1 / n)}\left((t-x)^{2}, x\right)=x(1-x) f^{\prime \prime}(x) .
$$

Theorem 2 now follows from (9), (12), (13), (14) immediately.

Theorem 3 For $f \in C[0,1], x \in\left[\frac{1}{4}, \frac{3}{4}\right]$, when $n$ sufficient large, we have

$$
\left|\widehat{D}_{n}^{(1 / n)}(f, x)-f(x)\right| \leq 2 \omega\left(f, \sqrt{\frac{3 x(1-x)}{n}}\right) .
$$

Proof Using linearity and monotonicity of $\widehat{D}_{n}^{(1 / n)}(f, x)$ we easily get, for every $\lambda>0$, that

$$
\begin{aligned}
& \left|\widehat{D}_{n}^{(1 / n)}(f, x)-f(x)\right| \leq\left|\widehat{D}_{n}^{(1 / n)}(|f(t)-f(x)|, x)\right| \\
& \quad \leq \omega(f, \lambda)\left(1+\frac{1}{\lambda} \widehat{D}_{n}^{(1 / n)}(|t-x|, x)\right) \\
& \quad \leq \omega(f, \lambda)\left(1+\frac{1}{\lambda} \sqrt{\widehat{D}_{n}^{(1 / n)}\left((t-x)^{2}, x\right)}\right) .
\end{aligned}
$$

Choosing $\lambda=\sqrt{\widehat{D}_{n}^{(1 / n)}\left((t-x)^{2}, x\right)}$, then (15) follows from (8) immediately.

Theorem 4 For $f \in C[0,1], x \in\left[\frac{1}{4}, \frac{3}{4}\right]$, when $n$ sufficient large, there exists a constant $C>0$ such that

$$
\left|\widehat{D}_{n}^{(1 / n)}(f, x)-f(x)\right| \leq C \omega_{2}\left(f, \sqrt{\frac{3 x(1-x)}{2 n}}\right) .
$$

Proof Let $g \in W^{2}$. By Taylor's expansion, we get

$$
g(t)=g(x)+g^{\prime}(x)(t-x)+\int_{x}^{t}(t-u) g^{\prime \prime}(u) d u .
$$

From Lemma 3, we have

$\widehat{D}_{n}^{(1 / n)}(g, x)=g(x)+\widehat{D}_{n}^{(1 / n)}\left(\int_{x}^{t}(t-u) g^{\prime \prime}(u) d u, x\right)$,

Hence

$$
\left|\widehat{D}_{n}^{(1 / n)}(g, x)-g(x)\right|
$$




$$
\begin{aligned}
& =\left|\widehat{D}_{n}^{(1 / n)}\left(\int_{x}^{t}(t-u) g^{\prime \prime}(u) d u, x\right)\right| \\
& \leq \widehat{D}_{n}^{(1 / n)}\left(\int_{x}^{t}(t-u)\left|g^{\prime \prime}(u)\right| d u, x\right) \\
& \leq \widehat{D}_{n}^{(1 / n)}\left((t-x)^{2}, x\right)\left\|g^{\prime \prime}\right\| \\
& \leq \frac{3 x(1-x)}{n}\left\|g^{\prime \prime}\right\| .
\end{aligned}
$$

Thus

$$
\begin{gathered}
\left|\widehat{D}_{n}^{(1 / n)}(f, x)-f(x)\right| \\
\leq\left|\widehat{D}_{n}^{(1 / n)}(f-g, x)\right|+|f-g|+\left|\widehat{D}_{n}^{(1 / n)}(g, x)-g(x)\right| \\
\leq 2|| f-g\left\|+\frac{3 x(1-x)}{n}\right\| g^{\prime \prime} \| .
\end{gathered}
$$

Taking the infimum on the right hand side over all $g \in W^{2}$, we obtain

$$
\left|\widehat{D}_{n}^{(1 / n)}(f, x)-f(x)\right| \leq 2 K_{2}\left(f, \frac{3 x(1-x)}{2 n}\right),
$$

By Lemma 5, we get

$$
\left|\widehat{D}_{n}^{(1 / n)}(f, x)-f(x)\right| \leq C \omega_{2}\left(f, \sqrt{\frac{3 x(1-x)}{2 n}}\right) .
$$

Theorem 5 For $f \in C[0,1], x \in\left[\frac{1}{4}, \frac{3}{4}\right]$ and $\phi(x)=\sqrt{x(1-x)}$, when $n$ sufficient large, there exists a constant $C>0$ such that

$$
\left|\widehat{D}_{n}^{(1 / n)}(f, x)-f(x)\right| \leq C \omega_{\phi}\left(f, \sqrt{\frac{6}{n}}\right) .
$$

Proof Using the representation

$$
g(t)=g(x)+\int_{x}^{t} g^{\prime}(u) d u
$$

we get

$$
\widehat{D}_{n}^{(1 / n)}(g, x)=g(x)+\widehat{D}_{n}^{(1 / n)}\left(\int_{x}^{t} g^{\prime}(u) d u, x\right),
$$

For any $x, t \in(0,1)$, we find that

$$
\left|\int_{x}^{t} g^{\prime}(u) d u\right| \leq\left\|\phi g^{\prime}\right\|\left|\int_{x}^{t} \frac{1}{\phi(u)} d u\right| .
$$

But

$$
\left|\int_{x}^{t} \frac{1}{\phi(u)} d u\right|=\left|\int_{x}^{t} \frac{1}{\sqrt{u(1-u)}} d u\right|
$$

$$
\begin{aligned}
& \leq\left|\int_{x}^{t}\left(\frac{1}{\sqrt{u}}+\frac{1}{\sqrt{1-u}}\right) d u\right| \\
& \leq 2(|\sqrt{t}-\sqrt{x}|+|\sqrt{1-t}-\sqrt{1-x}|) \\
& =2|t-x|\left(\frac{1}{\sqrt{t}+\sqrt{x}}+\frac{1}{\sqrt{1-t}+\sqrt{1-x}}\right) \\
& \leq 2|t-x|\left(\frac{1}{\sqrt{x}}+\frac{1}{\sqrt{1-x}}\right) \leq \frac{2 \sqrt{2}|t-x|}{\phi(x)} .
\end{aligned}
$$

Using Cauchy-Schwarz inequality, we obtain

$$
\left|\widehat{D}_{n}^{(1 / n)}(g, x)-g(x)\right|
$$

$$
\begin{aligned}
& \leq 2 \sqrt{2}\left\|\phi g^{\prime}\right\| \phi^{-1}(x) \widehat{D}_{n}^{(1 / n)}(|t-x|, x) \\
& \leq 2 \sqrt{2}\left\|\phi g^{\prime}\right\| \phi^{-1}(x)\left(\widehat{D}_{n}^{(1 / n)}\left((t-x)^{2}, x\right)\right)^{1 / 2} \\
& \leq \frac{2 \sqrt{6}\left\|\phi g^{\prime}\right\|}{\sqrt{n}} .
\end{aligned}
$$

Thus

$$
\begin{gathered}
\left|\widehat{D}_{n}^{(1 / n)}(f, x)-f(x)\right| \\
\leq\left|\widehat{D}_{n}^{(1 / n)}(f-g, x)\right|+|f-g|+\left|\widehat{D}_{n}^{(1 / n)}(g, x)-g(x)\right| \\
\leq 2\|f-g\|+\frac{2 \sqrt{6}\left\|\phi g^{\prime}\right\|}{\sqrt{n}} .
\end{gathered}
$$

Taking the infimum on the right hand side over all $g \in W_{\phi}[0,1]$, we obtain

$$
\left|\widehat{D}_{n}^{(1 / n)}(f, x)-f(x)\right| \leq 2 K_{\phi}\left(f, \sqrt{\frac{6}{n}}\right) .
$$

By Lemma 6, we get

$$
\left|\widehat{D}_{n}^{(1 / n)}(f, x)-f(x)\right| \leq C \omega_{\phi}\left(f, \sqrt{\frac{6}{n}}\right) .
$$

Theorem 6 For $f \in \operatorname{Lip}_{M}(\beta), x \in\left[\frac{1}{4}, \frac{3}{4}\right]$, when $n$ sufficient large, we have

$$
\left|\widehat{D}_{n}^{(1 / n)}(f, x)-f(x)\right| \leq M\left(\frac{3 x(1-x)}{n}\right)^{\beta / 2} .
$$

Proof By Hölder's inequality with $p=$ $\frac{2}{\beta}, q=\frac{2}{2-\beta}$, in view of Remark 2 we have

$$
\left|\widehat{D}_{n}^{(1 / n)}(f, x)-f(x)\right|
$$




$$
\begin{aligned}
& \leq \widehat{D}_{n}^{(1 / n)}(|f(t)-f(x)|, x) \\
& \leq M \widehat{D}_{n}^{(1 / n)}\left(|t-x|^{\beta}, x\right) \\
& \leq M\left(\widehat{D}_{n}^{(1 / n)}\left((t-x)^{2}, x\right)\right)^{\beta / 2} \\
& \leq M\left(\frac{3 x(1-x)}{n}\right)^{\beta / 2} .
\end{aligned}
$$

\section{Acknowledgment}

This work is supported by the Program for Outstanding Youth Scientific Research Talents in Fujian Province University(2016).

\section{References}

[1] L. Lupas and A. Lupas, Polynomials of binomial type and approximation operators, Stud Univ Babes-Bolyai Math, 1987, 32(4): 61-69.

[2] D. Miclaus, The revision of some results for Bernstein-Stancu type operators, Carpathian J Math, 2012, 28(2):289-300.

[3] V. Gupta and T.M. Rassias, LupasDurrmeyer operators based on polya distribution, Banach J Math Anal, 2014, 8(2): 146-155.

[4] P,N. Agrawal, N. Ispir and A. Kajla, Approximation properties of Lupas-Kantorovich operators based on polya distribution, Rendiconti del Circolo Matematico di Palermo Series 2, 2016, 65(2):185-208.

[5] J.P. King, Positive linear operators which preserve $x^{2}$, Acta Math Hungar, 2003, 99(3): 203-208.

[6] O. Duman and M.A. Ozarslan, SzaszMirakjan type operators providing a better error estimation, Appl Math Lett, 2007, 20(12): 1184-1188.

[7] L. Rempulska and K. Tomczak, Approximation by certain linear operators preserving $x^{2}$, Turkish J Math, 2009, 33(3): 273-281.

[8] A. Aral and V. Gupta, Applications of $q$ calculus in operator theory, Springer, New York, 2013.

[9] Z. Ditzian and V. Totik, Moduli of Smoothness, Springer, New York, 1987.

[10] R.A. Devore and G.G. Lorentz, Construtive Approximation, Springer-Verlag, Berlin, 1993. 